\title{
Three-dimensional Tomography of the Galactic and Extragalactic Magnetoionic Medium with the SKA
}

\author{
J. L. Han ${ }^{* 1}$, W. van Straten ${ }^{2}$, T. J. W. Lazio ${ }^{3}$, A. Deller ${ }^{4}$, C. Sobey ${ }^{4}$, J. Xu1, \\ D. Schnitzeler ${ }^{5}$, H. Imai ${ }^{6}$, S. Chatterjee ${ }^{7}$, J.-P. Macquart ${ }^{8}$, M. Kramer ${ }^{5}$, J. Cordes ${ }^{7}$ \\ ${ }^{1}$ National Astronomical Observatories, Chinese Academy of Sciences, Beijing 100012, China; \\ ${ }^{2}$ Swinburne University of Technology, Australia; ${ }^{3}$ Jet Propulsion Laboratory, California Institute \\ of Technology, USA; ${ }^{4}$ ASTRON, The Netherlands; ${ }^{5}$ MPIfR, Germany; ${ }^{6}$ Kagoshima University, \\ Japan; ${ }^{7}$ Cornell University, USA; ${ }^{8}$ Curtin University, Australia. \\ E-mail: hjlebao.ac.cn
}

The magneto-ionic structures of the interstellar medium of the Milky Way and the intergalactic medium are still poorly understood, especially at distances larger than a few kiloparsecs from the Sun. The three-dimensional (3D) structure of the Galactic magnetic field and electron density distribution may be probed through observations of radio pulsars, primarily owing to their compact nature, high velocities, and highly-polarized short-duration radio pulses. Phase 1 of the SKA, i.e. SKA1, will increase the known pulsar population by an order of magnitude, and the full SKA, i.e. SKA2, will discover pulsars in the most distant regions of our Galaxy. SKA1-VLBI will produce model-independent distances to a large number of pulsars, and wide-band polarization observations by SKA1-LOW and SKA1-MID will yield high precision dispersion measure, scattering measure, and rotation measure estimates along thousands of lines of sight. When combined, these observations will enable detailed tomography of the large-scale magneto-ionic structure of both the Galactic disk and the Galactic halo. Turbulence in the interstellar medium can be studied through the variations of these observables and the dynamic spectra of pulsar flux densities. SKA1-LOW and SKA1-MID will monitor interstellar weather and produce sensitive dynamic and secondary spectra of pulsar scintillation, which can be used to make speckle images of the ISM, study turbulence on scales between $\sim 10^{8}$ and $10^{13} \mathrm{~m}$, and probe pulsar emission regions on scales down to $\sim 10 \mathrm{~km}$. In addition, extragalactic pulsars or fast radio bursts to be discovered by SKA1 and SKA2 can be used to probe the electron density distribution and magnetic fields in the intergalactic medium beyond the Milky Way.

Advancing Astrophysics with the Square Kilometre Array

8-13 June 2014

Giardini Naxos, Italy

${ }^{*}$ Speaker. 


\section{Introduction}

Our Milky Way consists of the Galactic disk, which has a few spiral arms, the extended Galactic halo, the Galactic bulge, and a central bar. However, a detailed picture of the Milky Way is not yet clear. Spiral structures have been observed only in the closest half of our Milky Way, and the pitch angles of only a few arm segments have been determined (see Hou \& Han 2014). Embedded in these large-scale structures, the interstellar medium (ISM) consists of diffuse neutral hydrogen (H I) gas and H I clouds, higher density regions of molecular clouds, diffuse ionized gas (H II) and extended or compact H II regions. In turn, the ISM is permeated by magnetic fields, and the magnetized interstellar plasma is known as the magnetoionic medium. Although the magnetoionic medium occupies a significant fraction $(\sim 0.2)$ of the volume of the ISM, we have only limited knowledge of its distribution in the disk and halo. A considerable amount of energy is required to keep the ISM ionized, roughly $15-20 \%$ of the luminosity of all O/B stars in the Galaxy. Accordingly, ionized gas traces the energy input into the ISM, which is dominated by supernova remnants and the stellar winds of bright young stars. Therefore, in the Galactic disk, more ionized gas and more disturbed small-scale structures are expected to be associated with the spiral arms (as opposed to the interarm regions). However, the origin of hot gas in the Galactic halo, where very few hot stars exist, is not clear.

Magnetic fields are ubiquitous throughout the Universe. The magnetic fields in our Galaxy play a crucial role in numerous astrophysical processes: they affect the propagation of cosmic rays, impact the evolution of molecular clouds and star formation, and facilitate the transport of heat, angular momentum and energy. Measurements of the magnetic structure of the Milky Way are sparse, especially at distances greater than several kiloparsecs from the Sun and on length scales shorter than tens of parsecs. Zeeman splitting measurements probe the in situ magnetic fields of small-scale star formation regions, which are evidently related to the large scale structure of the Galactic magnetic field (Han \& Zhang 2007; see also Green et al. 2015 and Robishaw et al. 2015 in this volume).

Faraday rotation measurements probe the integrated magnetic field along the line of sight to pulsars and extragalactic radio sources. Because pulsars are distributed throughout the Milky Way, their compact nature and short-duration, highly-polarized radio pulses make them ideal probes of the three-dimensional structure of the diffuse magnetoionic medium in our Galaxy. Extraction of the common contribution to Faraday rotation measures of extragalactic radio sources yields the total Faraday rotation due to the Galactic magnetoionic medium (Xu \& Han 2014a,b; Haverkorn et al. 2015 in this volume).

To date, 2300 pulsars have been discovered using single dish telescopes (see the updated cata$\log$ of Manchester et al. 2005). Most of the known pulsars are in the Galactic disk, and about 20\% of them are in the Galactic halo (see Figure 1). The distribution of pulsars at low Galactic latitudes occupies about half of the Galactic disk; at high latitudes, the distribution is limited to about $4 \mathrm{kpc}$ from the Sun. A large spatial volume of the Milky Way is left for future pulsar discovery by the next generation of radio telescopes with extremely high sensitivity, such as FAST (Nan et al. 2011), LOFAR (Coenen et al. 2014) and the SKA (Keane et al. 2015).

According to the current SKA1 Baseline Design (Dewdney et al. 2013), Phase 1 of the SKA will consist of three arrays: a low-frequency array (SKA1-LOW), a mid-frequency array (SKA1- 

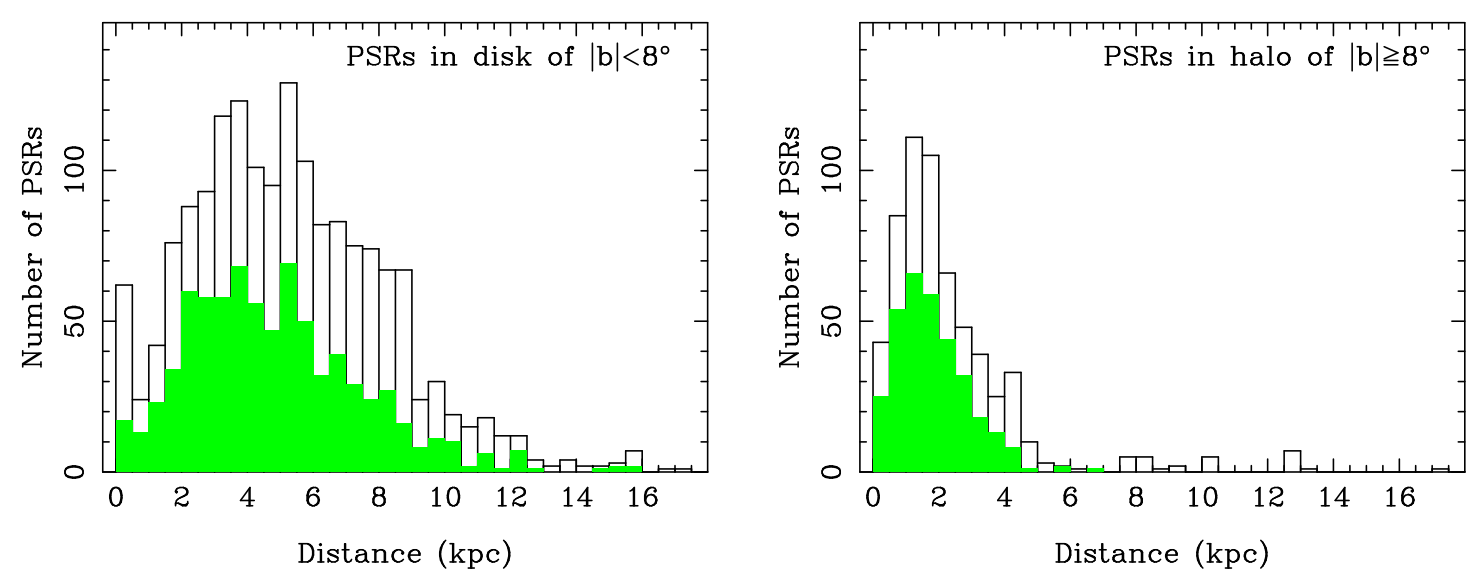

Figure 1: Distance distribution of known pulsars in the Galactic disk $\left(|b|<8^{\circ}\right)$ and in the Galactic halo $|b| \geq 8^{\circ}$. The green histograms correspond to pulsars with available RMs.

MID), and a wide-field survey array (SKA1-SUR). SKA1-LOW will be able to observe the $-67^{\circ}<$ Dec $<13^{\circ}$ sky in the frequency range of $50-350 \mathrm{MHz}$. The core array with a maximum baseline of $50 \mathrm{~km}$ will have a resolution of $11^{\prime \prime}$, and its collecting area of $800,000 \mathrm{~m}^{2}$ will reach a flux density limit of $2 \mu \mathrm{Jy} \mathrm{hr}^{-1 / 2}$. In 10-min integrations, the $600 \mathrm{~m}$ core array can reach $0.05 \mathrm{mJy}$. It is predicted that SKA1-LOW will find about 7,000 normal pulsars and about 900 millisecond pulsars (Keane et al. 2015), mostly in the closest half of the Galactic halo. SKA1-MID will operate in 5 different frequency bands; of relevance to this study are the three bands that overlap with those of SKA1-SUR: band-1: 350-1,050 MHz (MID) or 350-900 MHz (SUR); band-2: 950-1,760 MHz (MID) or 650-1,670 MHz (SUR); and band-3: 1,650-3,050 MHz (MID) or 1,500-4,000 MHz (SUR). Band-1 will be used to survey the halo pulsars at high Galactic latitudes, band-2 will be used at mid latitudes and to reach pulsars in the furthest reaches of the Galactic disk, and band-3 will be used at very low latitudes (e.g. $|b|<10^{\circ}$ ). According to simulations, SKA1-MID will find about 9,000 normal pulsars and about 1400 millisecond pulsars (see Fig. 3 of Keane et al. 2015) distributed in about $2 / 3$ of the Galactic disk and reaching $5 \mathrm{kpc}$ farther than the Galactic center. With approximately $50 \%$ of the sensitivity of SKA1, the early phase SKA1 will discover only $1 / 4$ or $1 / 3$ of the above predicted numbers of pulsars (assuming that similar surveys are done). SKA2LOW is expected to discover 11,000 pulsars, including 1500 millisecond pulsars, and SKA2-MID will find 24,000 - 30,000 pulsars, of which 2,400 - 3,000 will be millisecond pulsars. The most distant pulsars will be about $10 \mathrm{kpc}$ beyond the Galactic center in the other half of the disk.

The large numbers of pulsars discovered by SKA1 and SKA2 can be used to constrain the structure of the magnetoionic medium with unprecedented detail. Using pulsar dispersion measure (DM) and scattering measure (SM) estimates, combined with parallax distance estimates, we will construct the three-dimensional electron density distribution of the Milky Way, especially the poorly-explored region around the Galactic center and in the halo, as discussed in Section 2. Furthermore, the small-scale structure of the magnetoionic medium will be illuminated by high quality scintillation data and the dynamic spectra of many bright pulsars. Pulsar Faraday rotation measure (RM) estimates, combined with pulsar DM estimates, will be used to reveal the detailed three-dimensional structure of the Galactic magnetic field, as shown in Section 3. In fact, the high 
sensitivity of SKA1 and SKA2 should also enable the discovery of a large number of pulsars in nearby galaxies, which opens the window for the study of the intergalactic medium (IGM), as discussed in Section 4. The conclusions of this chapter are presented in Section 5.

\section{SKA1 for electron density distribution}

A better understanding of the electron-density distribution is crucial for estimating distances to large numbers of distant pulsars and deriving the magnetic field from Faraday rotation measurements (see Sect. 3). The ionized gas in the ISM is not uniformly distributed. In the Galactic disk, high density clumps are associated with $\mathrm{H}$ II regions and bright stars, such that the distribution of higher density ionized gas follows the spiral arms. There are also many voids that are associated with bubbles or superbubbles. The ionized gas can be directly imaged by $\mathrm{H} \alpha$ surveys (Finkbeiner 2003), but its distribution throughout the Galactic disk is difficult to ascertain owing to our location near the edge of the Galactic disk. Measurements of ionized gas in the Galactic halo and in the area around the Galactic center are limited.

\subsection{Pulsar distances and the electron density distribution}

The dispersion measure (DM) of a pulsar is the integral of the free electron density between the pulsar and us, DM $=\int_{\mathrm{us}}^{\mathrm{PSR}} n_{e} d l$. The DMs of a large number of widely distributed pulsars can be used to construct a model of the electron density distribution, as long as the distances to these pulsars can be independently measured. However, pulsar distances are difficult to measure. Available methods include 1) measuring annual parallax using Very Long Baseline Interferometry (VLBI) astrometry, pulsar timing, or even by direct multi-epoch high resolution optical images; 2) establishing an association between a pulsar and a supernova remnant (SNR) that has an estimated distance; 3) observing HI absorption and deriving the kinematic distance with the Galactic rotation curve. Only with an accurate distance can a pulsar dispersion measure be converted to an average electron density along the line of sight, and a large number of such sight-lines (of different length and in different directions) are necessary to construct a suitably detailed model of the ionized ISM of the Milky Way.

Currently, the most widely used model for the electron density distribution in the Milky Way is the NE2001 model (Cordes and Lazio 2002; see the yellow background in Fig. 3), which has received over 800 citations and is an essential reference model for Galactic studies. At the time it was constructed, distance estimates were available for $\sim 100$ pulsars: 19 via parallax (13 via VLBI, 5 via timing and 1 via optical imaging), 74 via HI absorption, 8 via SNR associations, 16 via globular cluster associations, and 8 pulsars in the Magellanic clouds.

In order to improve our understanding of the ionized ISM, it is crucial to accurately estimate the distance to as many pulsars as possible. Since NE2001 was finalized, a wealth of new information on pulsar distances has been obtained, with the number of measured parallaxes ${ }^{1}$ increasing five-fold. In particular, the number of precise measurements made with Very Long Baseline Interferometry has exploded, predominantly due to careful work with the Very Long Baseline Array (Brisken et al. 2002; Chatterjee et al. 2009; Deller et al. 2011). Using pulsars at known distances,

\footnotetext{
${ }^{1}$ see e.g. http://www.astro.cornell.edu/research/parallax/
} 
which come from either parallax measurements or by association with Galactic globular clusters or the Magellanic Clouds, Gaensler et al. (2008) found that the ionized thick disk in the Milky Way could have a scale height of $1.8_{-0.2}^{+0.1} \mathrm{kpc}$, which is almost a factor of two larger than the scale height used in NE2001 and its predecessor, the TC93 model by Taylor \& Cordes (1993). By also including other model components from TC93 and NE2001 when fitting for the scale height of the thick disk, Schnitzeler (2012) subsequently showed that the difference in scale height is probably not so large. These results demonstrate that measuring geometric parallaxes for a large sample of pulsars has the potential to considerably change our understanding of the structure of the ionized Milky Way. A revised "NE2014" model incorporating the latest distance and scattering constraints would already form a considerable advance in our knowledge of the ionized ISM.

However, despite the recent advances in VLBI precision (where parallax errors of 0.02 mas and better are now attainable; see e.g. Deller et al. 2013), most pulsars with a geometric distance measurement are still in the local Galactic neighbourhood; the existing VLBI and timing parallaxes span 6.4 to $0.2 \mathrm{mas}$, and the median of 64 distances measured by parallax is just $\sim 0.9 \mathrm{kpc}$. To make a truly Galactic-scale model, many new constraints at larger distances are required.

Fortunately, in addition to discovering many new pulsars, the SKA will also excel in the measurement of their distances. Precision timing of millisecond pulsars with SKA1 will yield a significant number of timing parallax distances widely distributed across the Galaxy (Keane et al. 2015). The exact number will depend on the observing time allocated to pulsar timing programs, but observations for pulsar timing arrays (Janssen et al. 2015 in this volume) will ensure a moderately sized sample.

SKA1-VLBI observations (in which SKA1-mid and SKA1-survey are used as sensitive phasedarray elements in a VLBI array; Paragi et al. 2015) will provide even greater parallax precision, capable of measuring distances across the Galaxy. This technique will also be more widely applicable because all radio pulsars can be targeted, not only millisecond pulsars. Observing time will likely limit the number of sources for which VLBI parallaxes can be obtained with SKA1, but it will remain possible to obtain a sample of sources that cover a representative region of the Galaxy. Both timing and VLBI parallaxes will be possible with early-phase SKA1 at 50\% sensitivity, although in each case they will be unable to access the faintest members of the population.

With SKA2, the situation improves further. The increase in collecting area will make even higher precision timing parallaxes possible for fainter sources; for imaging observations, the addition of several thousand $\mathrm{km}$ baselines to the SKA will make VLBI-style observations possible with SKA2 alone. Smits et al. (2011) estimated that of order 10,000 pulsar distances could be measured by SKA2, with the majority obtained via imaging observations.

\subsection{Wide-band observations of pulsar scattering}

The emission region of pulsars is very small (less than a few hundred kilometers), such that pulsars are effectively point radio sources (with angular sizes of nanoarcseconds). When pulsed radio emission from a point source passes through the inhomogeneous interstellar plasma, two manifestations of multi-path propagation effects can be observed: pulse broadening in the time domain and a scattering disk in the image domain. With the SKA, the inhomogeneous interstellar plasma will produce observable effects on lines of sight to thousands of pulsars. These effects will be most significant in the SKA1-LOW band, and combination with the wide frequency range in the 


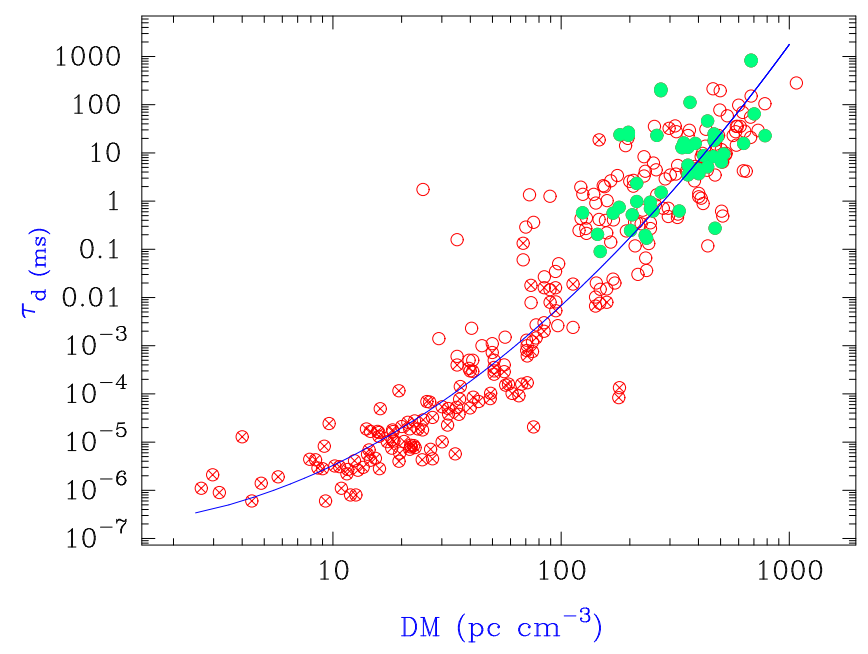

Figure 2: The time scales of pulse broadening are related to pulsar DM, in addition to the frequency dependence $v^{-\alpha}$ (see Bhat et al. 2004 for details: the filled dots denote measurements from multifrequency profiles presented in that paper, crossed circles denote measurements based on pulsar decorrelation bandwidths, and open circles denote previous measurements in the literature. The line is the best fit of equation 2.1 to the data).

two lower bands of SKA1-MID and SKA1-SUR will enable the unprecedented use of interstellar scattering to study the properties of the intervening medium.

To date, pulse broadening has been measured at several frequencies for only about 150 pulsars (e.g. Löhmer et al. 2001, 2004; Bhat et al. 2004; Lewandowski et al. 2013). For nearby pulsars (within a few kpc) the pulse broadening time scale varies with radio frequency as $\tau \sim v^{-\alpha}$, where $\alpha \sim 4.4$, which is consistent with a Kolmogorov spectrum of electron density fluctuations. For distant pulsars, the $\alpha$ values deviate from a single thin screen model. Because ISM irregularities are associated with spiral arms, the most distant pulsars with sight-lines passing through several spiral arms should exhibit very different scattering behaviour that is not as simple as the Kolmogorov spectrum (Lewandowski et al. 2013). The time scale of pulse broadening is related to the pulsar DM by

$$
\log \tau \simeq a+b(\log \mathrm{DM})+c(\log \mathrm{DM})^{2}-\alpha \log v
$$

(see Fig.2 of Bhat et al. 2004) with $a=-6.46, b=0.154$ and $c=1.07$. The estimated value of $\alpha=3.86 \pm 0.16$ is significantly lower than the value of 4.4 that is appropriate for a Kolmogorov medium. However, the dispersion of $\tau$ estimates spans one or two orders of magnitude in Fig. 2. Some pulsars with high DMs do not show strong scattering as expected, such as PSR B2002+31 (Lyne 1971) and the Galactic center pulsar PSR J1745-2900 (Spitler et al. 2014b). This probably indicates a predominantly uniform medium along these lines of sight. It is possible that the interstellar plasmas in the Galactic halo and near the Galactic center have fewer irregularities and are more uniformly distributed than in the disk.

The scattering disks of only a few pulsars have been observed to date (e.g. Lazio 2004). The angular diameter of the disk depends on radio frequency as $\theta \sim v^{-\beta}$, where $\beta=-2.2$ for an electron density distribution described by a Kolmogorov spectrum. Note that angular broadening measurements often indicate $\beta \sim 2.0$ (e.g. at the Galactic Centre) and decorrelation bandwidths 
often scale as $v^{-4}$, both of which indicate that the diffractive scale probed by pulsar observations is within the inner scale of the turbulent cascade in the ISM. SKA-VLBI (Paragi et al. 2015) will provide the sensitivity, wide frequency coverage, high resolution, and high dynamic range required to overcome the technical challenges associated with observations of angular broadening.

Scattering in the ISM operates as an interferometer whose maximum baseline is of order the size of the scattering disk, up to tens of AU (e.g. Pen \& Levin 2014; Pen et al. 2014). Pulsar longitude-resolved SKA-VLBI observations at low frequencies can constrain source structure on nanoarcsecond scales, which will enable us to image pulsar magnetospheres on scales down to $\sim 10 \mathrm{~km}$; for more details, see Karastergiou et al. (2015) in this volume.

\subsection{Wide-band observations of pulsar scintillation}

Owing to the inhomogeneity of the ISM and the high relative velocities of neutron stars, pulsars exhibit flux density modulations as a function of radio frequency and time, known as scintillation. To characterize the diffractive scintillation, dynamic scintillation spectra are analyzed using a two-dimensional autocorrelation function to obtain the temporal and spectral scales of decorrelation, which are related to the diameter of scattering disk. Small-scale structures in the ISM cause rapid intensity variations (diffractive scintillation) and large-scale density structures cause slow refractive scintillation.

Very high sensitivity is required to observe the dynamic spectrum of a pulsar, which must be detectable in a narrow band in a short integration time. Accordingly, dynamic spectra have been observed for only the brightest pulsars (e.g. Gupta et al. 1994). Owing primarily to the high velocities of pulsars, the differences between dynamic spectra observed at different epochs probe the irregularities of the ISM on scales ranging of $\sim 10^{8}$ to $10^{13} \mathrm{~m}$.

Dynamic spectra with high signal-to-noise ratio $(S / N)$ can be further analyzed via the twodimensional Fourier transformation to yield the power spectrum of the dynamic spectrum, known as the secondary spectrum. Secondary spectra typically exhibit power concentrated in parabolic arcs, and each point on this arc arises from interference between points in the scattered image of the source. The parabolic shape arises from differential Doppler shift and differential delay between points in the scattered image, which vary linearly and quadratically, respectively, with scattering angle (e.g. Cordes et al. 2006). Features in the secondary spectra also vary as a function of radio frequency and observing epoch, see e.g. figures 1, 2 and 3 in Cordes et al. (2006) and figure 1 of Stinebring (2006). Multiple parabolic arcs are likely produced by multiple scattering screens along the line of sight.

The best observations of secondary spectra have been carried out at Arecibo (e.g. Cordes et al. 2006). Using the core array of SKA1-LOW and SKA1-MID, high $S / N$ dynamic and secondary scintillation spectra can be obtained for many pulsars in a wide-range of low frequencies; these can be used to study both the static and dynamic structure of the ISM.

\subsection{Interstellar weather}

The large collecting area and large fractional bandwidth at low frequencies of SKA-LOW combined with bands 1 and 2 of SKA-MID or SKA-SUR (including SKA1 and the early phase of SKA1) will provide very accurate DM and RM estimates for thousands of known and newly 
discovered pulsars. Owing to the $\lambda^{2}$ dependence of dispersive and refractive effects in the ISM, DM and RM estimates are best made at lower frequencies using wider bandwidths, as long as the pulse profile is not adversely affected by scattering.

Monitoring DM variations over time probes the structure of the ionized gas on length scales of the order of $10^{8}-10^{13} \mathrm{~m}$. The time scale for DM variations depends on the velocity of the pulsar and the characteristic size of clouds or filaments of ionized gas along the line of sight. Increased sensitivity enables the detection of DM variations on short time intervals due to small-scale clouds. By monitoring short-term DM variations along large numbers of pulsar lines of sight, SKA1 will probe the physical processes acting within the interstellar plasma that generate or maintain sub-AU density fluctuations.

Monitoring long-term DM variations reveals large clumps of ionized gas drifting across the lines of sight. Such observations have been made at Parkes using timing array pulsars over several years (You et al. 2007; Keith et al. 2013) and a sample of 168 young pulsars over 6 years (Petroff et al. 2013). DM and RM variations of pulsars near the ecliptic plane can also be used to study the solar wind (You et al. 2012). Note, however, that correcting ionospheric Faraday rotation is essential for such experiments (e.g. Sotomayor-Beltran et al. 2013).

\section{Interstellar magnetic fields}

Pulsars are ideal probes of interstellar magnetic fields for the following four reasons: 1) owing to the high degree of linear polarization of pulsar signals, the RM is easily measured; 2) intrinsic Faraday rotation in the pulsar magnetosphere is negligible, such that the observed Faraday rotation is completely due to the interstellar magnetoionic medium (after correcting for ionospheric Faraday rotation); 3) the pulsar DM provides the integrated free electron column density, allowing the lineof-sight magnetic field to be decoupled (Eq. 3.1); and 4) there are a large number of pulsars distributed throughout the Galaxy in both the disk and the halo, facilitating a three-dimensional picture of the Galactic electron density and magnetic field. For a pulsar at distance $D$ (in pc), the $\mathrm{RM}$ is given by $\mathrm{RM}=0.810 \int_{0}^{D} n_{e} \mathbf{B} \cdot d \mathbf{l}$, (in $\mathrm{rad} \mathrm{m}^{-2}$ ). With the pulsar dispersion measure, $\mathrm{DM}=\int_{0}^{D} n_{e} d l$, (in pc $\mathrm{cm}^{-3}$ ), we obtain a direct estimate of the magnetic field strength parallel to the line of sight, $\left\langle B_{\|}\right\rangle$in $\mu \mathrm{G}$, weighted by the local free electron density:

$$
\left\langle B_{\|}\right\rangle=\frac{\int_{0}^{D} n_{e} \mathbf{B} \cdot d \mathbf{l}}{\int_{0}^{D} n_{e} d l}=1.232 \frac{\mathrm{RM}}{\mathrm{DM}} .
$$

When RM and DM data are available for multiple pulsars along similar lines of sight, e.g. one pulsar at $D_{0}$ and one at $D_{1}$, the variation of DM and RM with distance can be used to derive the field direction and field strength in the region between $D_{0}$ and $D_{1}$,

$$
\left\langle B_{\|}\right\rangle_{D_{1}-D_{0}}=1.232 \frac{\Delta \mathrm{RM}}{\Delta \mathrm{DM}}
$$

where $\left\langle B_{\|}\right\rangle_{D_{1}-D_{0}}$ is the mean line-of-sight field component, $\Delta \mathrm{RM}=\mathrm{RM}_{D_{1}}-\mathrm{RM}_{D_{0}}$ and $\Delta \mathrm{DM}=$ $\mathrm{DM}_{D_{1}}-\mathrm{DM}_{D_{0}}$. This derived field strength is not dependent on the electron density model, though the pulsar distances may have to be estimated from the electron density model if they have not been independently measured (e.g. by VLBI or pulsar timing). 


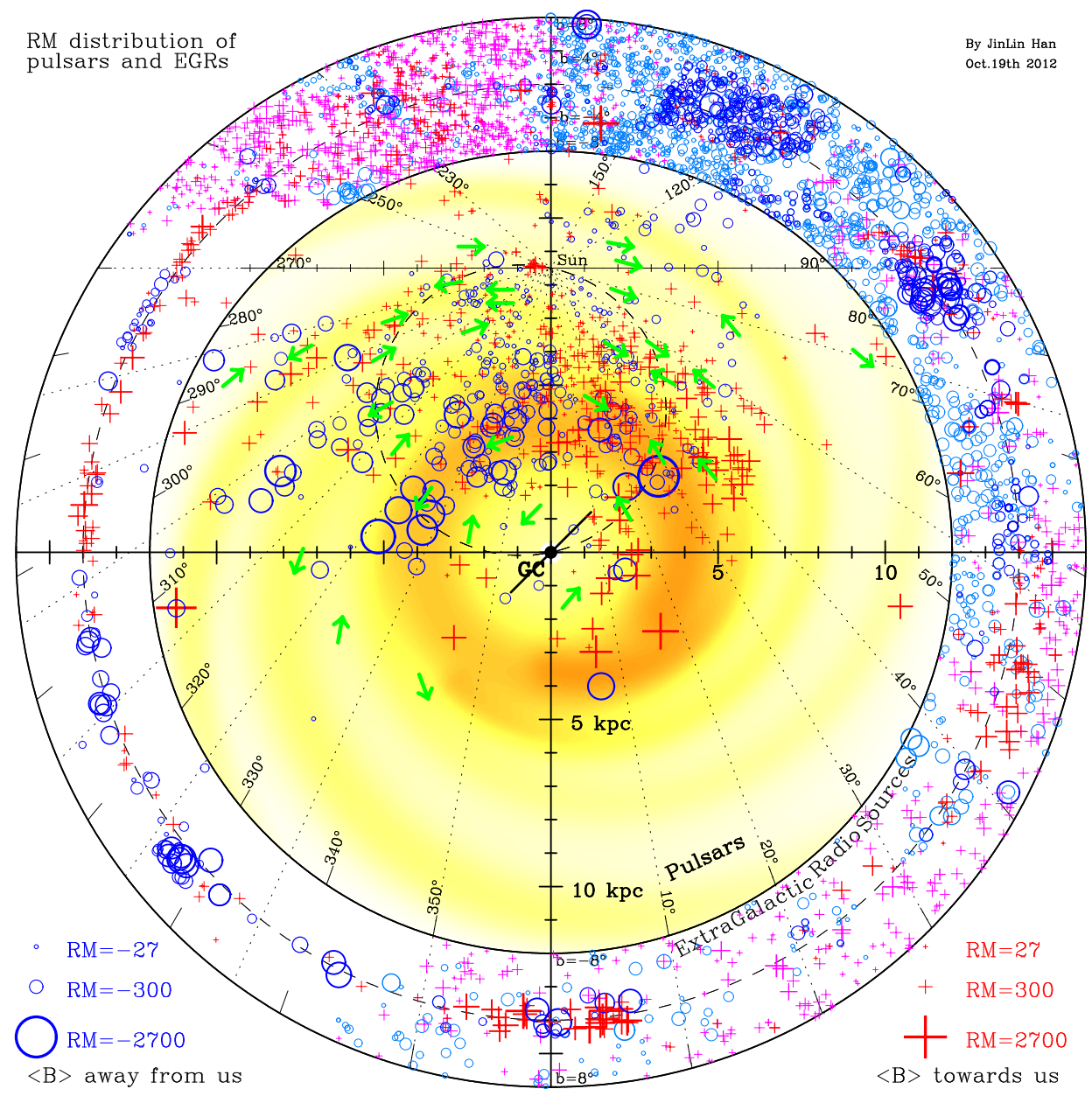

Figure 3: The RM distribution of 736 pulsars located within $|b|<8^{\circ}$ projected onto the Galactic plane. The background shows the approximate locations of spiral arms used in the NE2001 electron density model (Cordes \& Lazio 2002). RMs of extragalactic radio sources (Xu \& Han 2014b) located within $|b|<8^{\circ}$ are displayed in the outer ring according to their $l$ and $b$, with the same convention of RM symbols and limits. The derived large-scale structure of magnetic fields in the Galactic disk are indicated by arrows. See Han (2013) for details.

There are currently over 2,300 pulsars that have been discovered, and only $30 \%$ of these have published RM values. The RMs in the Galactic disk have been used to reveal the magnetic field structure in the closest half of the disk (Han et al. 2006). Over large scales, the magnetic fields follow the spiral arms; however, many field reversals are also observed (see Fig.3). It is clear that pulsar RM data are scarce in many regions of the closest half of the disk; there are only a few pulsar RM estimates in the most distant regions of the Galaxy. The distribution of RMs of extragalactic radio sources behind the Galactic disk (see Fig.3) can be used to derive the field structures beyond pulsars, and also can constrain the disk-field model (e.g. Sun et al. 2008).

The detailed structure of magnetic fields in the Galactic disk will be revealed by SKA1, which will discover several thousand pulsars in the closest half of the disk and measure their DMs and RMs, and SKA2, which will discover at least ten thousand pulsars in the most distant half of the disk (see Keane et al. 2015). Full-polarization observations of the newly discovered pulsars using 

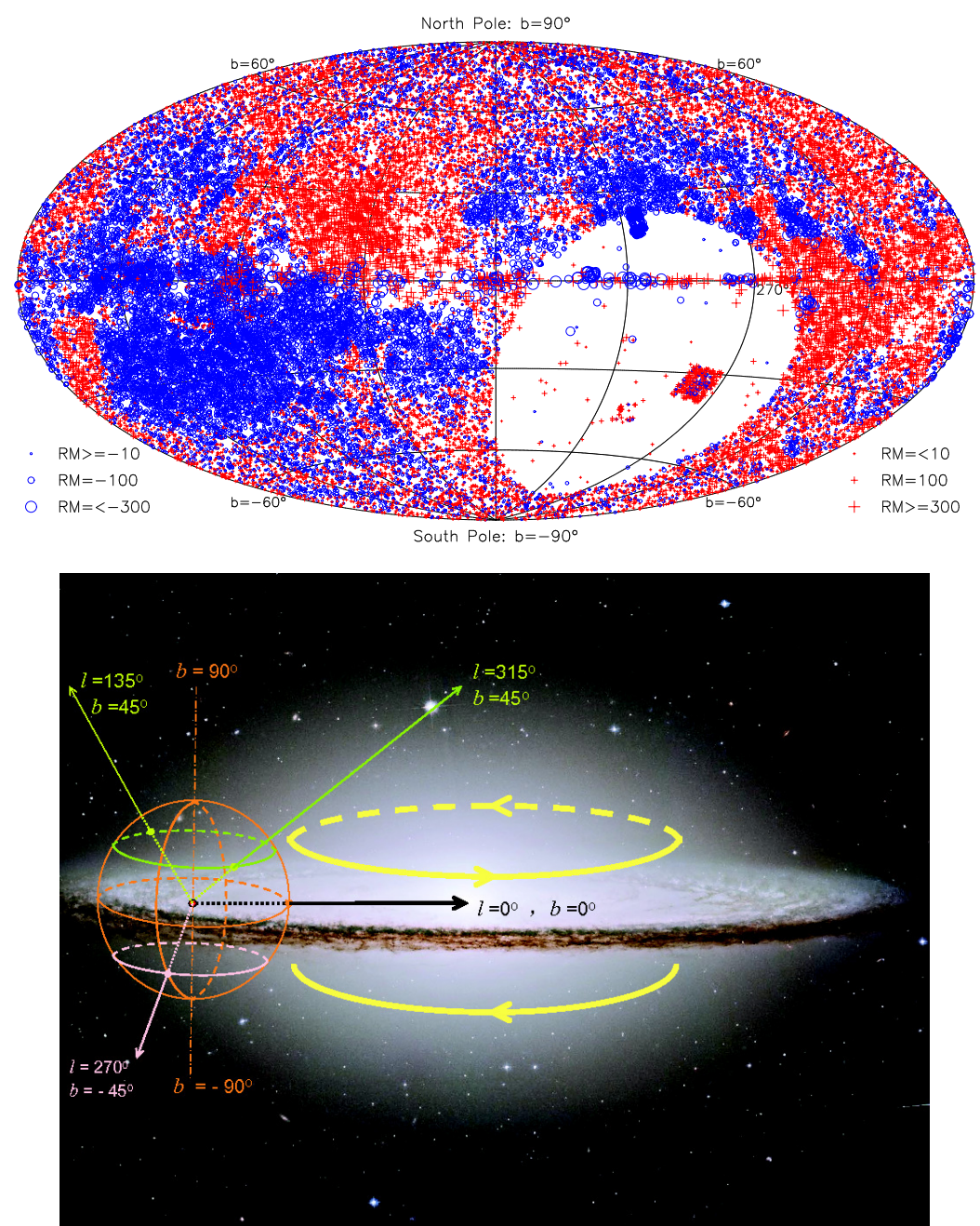

Figure 4: The sky distribution of RMs of extragalactic radio sources (top) and the Galactic coordinates and the azimuthal magnetic fields in the Galactic halo (bottom), see Han et al. $(1997,1999)$ and Xu \& Han (2014b). Currently, pulsar data are available only $\sim 3 \mathrm{kpc}$ from the Sun (see Fig.1). RMs of several thousand distant pulsars to be discovered by the SKA at the high latitudes are ultimate data to reveal the large-scale field structure in the Galactic halo.

SKA1-LOW (50 -350 MHz), and band 1 of SKA1-MID (350 -850 MHz) and SKA1-SUR (650$1,150 \mathrm{MHz}$ ) with high spectral resolution (up to 256,000 frequency channels in each band) will determine RMs with unprecedented precision $\left(\sim 0.1 \mathrm{rad} \mathrm{m}^{-2}\right)$ and constrain the detailed structure of the Galactic magnetoionic medium. Note, however, that such RM precision is only achievable if the ionospheric contribution is accurately corrected.

SKA1 and SKA2 will also complement the pulsar RM data by increasing the density of extragalactic radio sources with RM estimates. Some constraints on the large-scale structure of the Galactic magnetic field have been derived from the RMs of extragalactic radio sources, namely the striking antisymmetry in the inner Galaxy and the large-scale toroidal magnetic fields in the Galactic halo with reversed directions above and below the Galactic plane (see Fig.4). These structures were originally proposed by Han et al. $(1997,1999)$ and later modeled by other authors (e.g. Prouza 
\& Šmída 2003, Sun et al. 2008, Ferriére \& Terral 2014). However, the detailed properties of the halo magnetic field (e.g. the variation of field strength with radius and height) are not yet well constrained. Thousands of distant pulsars in the Galactic halo will be discovered by the SKA and their RMs will reveal the large-scale magnetic field structure in the Galactic halo.

In addition to the large-scale structure of the Galactic magnetic field, a variety of experiments probe small-scale structures in the interstellar magnetoionic medium. For example, compared to foreground pulsars along similar lines of sight, pulsars behind $\mathrm{H}$ II regions have significantly different Faraday rotation measures (e.g. Mitra et al. 2003). Evidence of similar small-scale fields associated with $\mathrm{H}$ II regions, supernova remnants and filaments has also been found in the RMs of extragalactic radio sources (e.g. Harvey-Smith et al. 2011; Sun et al. 2011; Savage et al. 2013). Such regions may be explored in greater detail by exploiting the increased density of extragalactic radio sources detected by SKA2, SKA1, and the early phase of SKA1.

Clearly, interstellar magnetic fields exist over a broad range of spatial scales, from large Galactic scales to very small dissipative scales. Determination of the magnetic energy spectrum offers a solid observational test for dynamo and other theories of Galactic magnetic field origin. The magnetic energy spectrum is currently constrained for only a small range of wavenumbers in relatively few regions. Magnetic fields on small spatial scales should follow the the Kolmogorov spectrum. Minter \& Spangler (1996) found that structure functions of rotation measure and emission measure were consistent with a Kolmogorov spectrum of three-dimensional turbulence in magnetic fields up to $4 \mathrm{pc}$, but with two-dimensional turbulence between $4 \mathrm{pc}$ and $80 \mathrm{pc}$. Using pulsar RMs in a large region of the Galactic disk, Han et al. (2004) obtained a power law distribution of magnetic field fluctuations described by $E_{B}(k)=C\left(k / \mathrm{kpc}^{-1}\right)^{-0.37 \pm 0.10}$ over spatial scales from $1 / k=0.5 \mathrm{kpc}$ to $15 \mathrm{kpc}$. An apparent turn over in the spectrum between $0.5 \mathrm{kpc}$ and $80 \mathrm{pc}$ is not yet clear (Han 2009). In combination with the RM spectra of intervening polarised sources, the dense grid of pulsars discovered with the SKA will probe the energy spectrum at least down to $100 \mathrm{pc}$ scales.

\section{The extragalactic magnetoionic medium}

In addition to significantly improving our understanding of the physics of the ISM in the Milky Way, the SKA will reveal the electron density distribution and magnetic field structure in nearby galaxies and the IGM. First, it will measure the DMs and RMs of extragalactic pulsars (especially their giant pulses) and single pulses of extragalactic origin, such as the Fast Radio Bursts (FRBs; Lorimer et al. 2007; Thornton et al. 2013, Spitler et al. 2014); see Macquart et al. (2015) in this volume for more details. Second, the SKA will yield high resolution observations of megamaser Zeeman splitting in nearby galaxies (e.g. Robishaw et al. 2008; McBridge \& Heiles 2013); for more details, see Robishaw et al. (2015) in this volume. Third, the SKA will observe the polarization of all radio sources in the sky over a wide band, yielding high precision RMs of a large number of distant radio sources shining through galaxies, clusters of galaxies and cosmic web in the nearby universe; see Heald et al. (2015), Johnston-Hollitt et al. (2015) and Beck et al. (2015) in this volume.

The high sensitivity, wide field of view, and wide bandwidth of SKA1 and SKA2 will yield a large sample of pulsars in nearby galaxies such as M31 and M33 (see Keane et al. 2015, this volume). At present, DMs of extragalactic objects have been estimated for only a handful of 

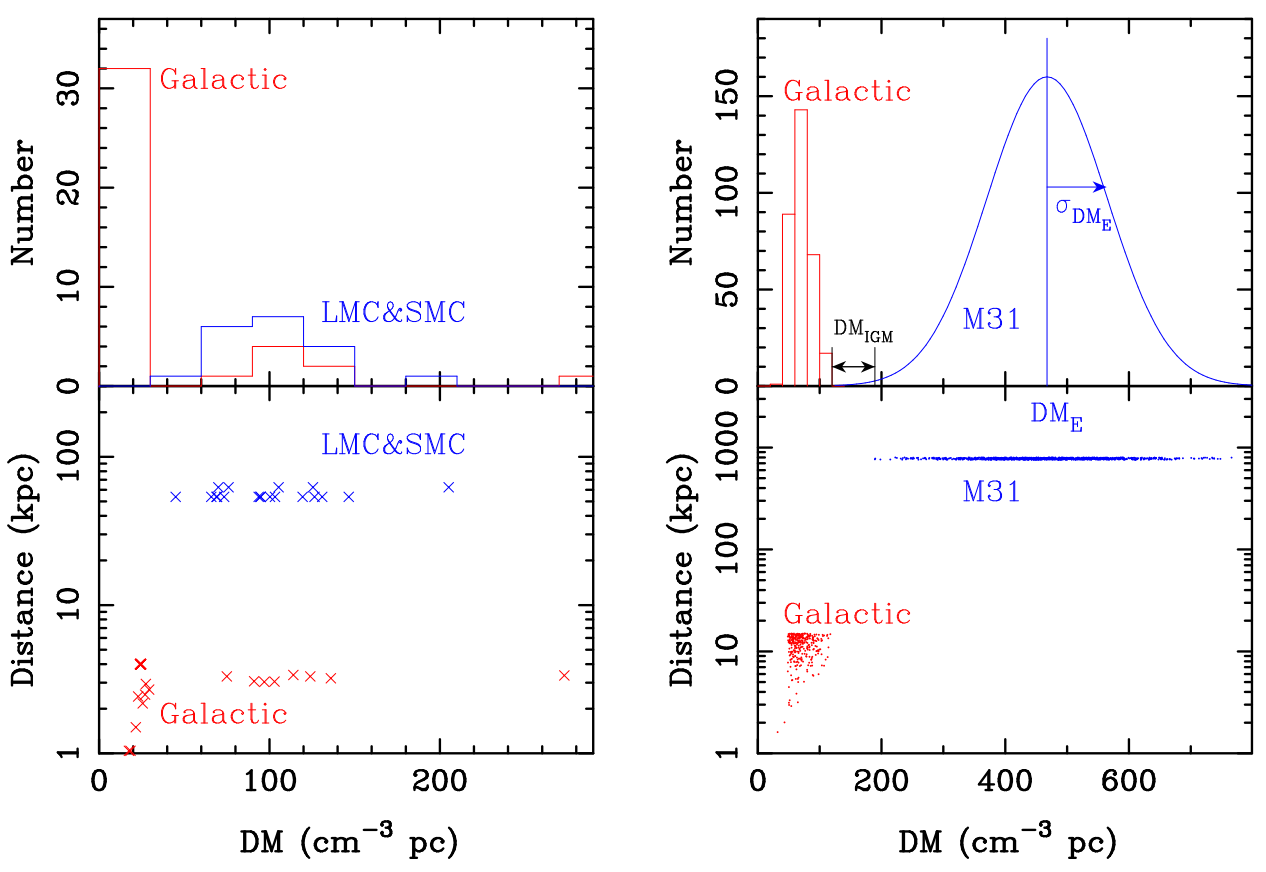

Figure 5: DM distributions of extragalactic pulsars (blue) and Galactic pulsars (red) within a $10^{\circ}$ radius around the host galaxy. Current data for pulsars in the large and small Magellanic Clouds are compared with Milky Way pulsars on the left and simulated data for pulsars in M31 are compared with simulated Milky Way pulsars on the right. Currently a small sample of pulsars in the Magellanic Clouds and a small sample of foreground Galactic pulsars around the clouds are not enough to deduce the intergalactic DM (or RM). However, the SKA will discover a much larger sample of Galactic and extragalactic pulsars (e.g. in M31) for this purpose. The DM range of extragalactic pulsars depends on the inclination angle of the host galaxy disk.

pulsars in the Magellanic Clouds (Manchester et al. 2006) and a few FRBs (Thornton et al. 2013). Scattering has been detected and studied for only four FRBs (e.g. Thornton et al. 2013); the RM of an FRB is yet to be observed.

Two key steps are needed to derive intergalactic DM and RM estimates from observations of extragalactic pulsars or pulses: 1) subtraction of the foreground DM or RM contribution from our Milky Way; and 2) constraining the local contribution from the host galaxy. Both steps require a large sample of pulsars. Estimating the foreground column density of electrons in the Milky Way requires a large sample of Galactic pulsars in the region of sky immediately around the direction of the host galaxy. To estimate the Galactic foreground contribution to the measured Faraday rotation also requires a large number of extragalactic radio sources around the direction of the host galaxy. SKA1 will observe a large number of extragalactic RMs as part of the cosmic magnetism project (e.g. Johnston-Hollitt et al. 2015); the average RM of background radio sources, $\left\langle\mathrm{RM}_{\mathrm{BGS}}\right\rangle$, in the direction of a host galaxy represents the foreground contribution of the Milky Way to the estimated RM of an extragalactic pulsar or pulse (e.g. see Xu \& Han 2014b).

For an individual extragalactic pulsar or pulse, it is difficult to estimate the dispersion and Faraday rotation contributed by the host galaxy. When a large sample of pulsars are discovered in a host galaxy, the minimum DM of these pulsars, $\min \left(\mathrm{DM}_{\mathrm{extraPSRs}}\right)$, can be used as the upper limit of $\mathrm{DM}_{\mathrm{IGM}}$ plus the Galactic DM foreground, $\max \left(\mathrm{DM}_{\text {GalacPSRs }}\right)$, as shown in Fig. 5. When 
averaged over a sample of extragalactic pulsars in the same host galaxy, the mean RM contributed by the host is likely to approach zero. Therefore, to probe the electron density and magnetic field of the IGM, we can estimate $\mathrm{DM}_{\mathrm{IGM}}=\min \left(\mathrm{DM}_{\mathrm{extraPSR}}\right)-\max \left(\mathrm{DM}_{\mathrm{GalacPRs}}\right)$ and $\mathrm{RM}_{\mathrm{IGM}}=$ $\left\langle\mathrm{RM}_{\mathrm{extraPSR}}\right\rangle-\left\langle\mathrm{RM}_{\mathrm{BGS}}\right\rangle$.

In addition to observing extragalactic pulsars and pulses, the SKA will be able to reveal extragalactic magnetism in a nearby galaxy by observing the RMs of either a large number of radio sources behind the galaxy or diffuse emission from the galaxy (see Heald et al. 2015). On the other hand, when a large sample of RMs of quasars or other objects with known redshifts are available (e.g. Johnston-Hollitt et al. 2015), the residual RMs (i.e. the values after subtracting the foreground RM from the observed RM values) can be used to explore the magnetoionic medium in the cosmic web and intervening clouds (see Xu \& Han 2014a). The distribution and statistics of residual RMs can reveal weak magnetic fields and their evolution over cosmological distances much greater than most detectable extragalactic pulsars.

\section{Conclusions}

Pulsars are the best probes of the interstellar magnetoionic medium in the Milky Way. By measuring dispersion toward a large number of pulsars and their distances, we can construct a detailed electron density distribution model. Scattering measurements derived from either the extended tails of pulsar profiles at low frequencies or from pulsar dynamic spectra will reveal the dynamics of small-scale structures in the ionized ISM. Faraday rotation probes the large and small scale structure of interstellar magnetic fields. Following the discussion in previous sections, we conclude the following:

- SKA1: Beginning with the early phase of SKA1, wide-band polarimetry with SKA1-LOW and SKA1-MID will provides a much better understanding of the structure of the interstellar medium. The DMs of several thousand newly discovered pulsars spread across the Milky Way will dramatically improve the electron density model, especially once independent and precise distances can be estimated for a substantial fraction of pulsars using both SKA1 pulsar timing and SKA1-VLBI. The distribution of pulsar RMs can be used to map out magnetic field structures in high detail.

- SKA2: By performing a complete census of those pulsars in our Galaxy that are visible in the southern hemisphere, and in concert with large telescopes in the northern hemisphere, SKA2 will complete the model of electron density distribution and magnetic field structure in the entire Milky Way,

- Intergalactic medium: SKA1 and SKA2 can be used to discover a large number of pulsars in nearby galaxies. By observing the DMs and RMs of a large sample of extragalactic pulsars and subtracting the Galactic foreground and host galaxy contributions, we will have the unique chance to detect the baryonic content and magnetic field of the intergalactic medium. The high sensitivity of the SKA is essential to this purpose. In addition, the statistics of RMs of a large number of extragalactic radio sources can reveal the weak magnetic fields in the cosmic web and other intervening galaxies. 


\section{Acknowledgments}

JinLin Han is supported by the National Natural Science Foundation (No.11473034) and by the Strategic Priority Research Program "The Emergence of Cosmological Structures" of the Chinese Academy of Sciences, Grant No. XDB09010200. The research of Joseph Lazio was performed at the Jet Propulsion Laboratory, California Institute of Technology, under a contract with the National Aeronautics and Space Administration.

\section{REFERENCES}

Beck, R., Bomans, D., Colafrancesco, S. et al., 2015, "Structure, dynamical impact and origin of magnetic fields in nearby galaxies in the SKA era", in proc. Advancing Astrophysics with the Square Kilometre Array, PoS(AASKA14)094

Bhat, N. D. Ramesh, Cordes, J. M., Camilo, F., et al. 2004, ApJ, 605, 759

Brisken, W. F., Benson, J. M., Goss, W. M., \& Thorsett, S. E. 2002, ApJ, 571, 906

Chatterjee, S., Brisken, W. F., Vlemmings, W. H. T., et al. 2009, ApJ, 698, 250

Coenen, T., van Leeuwen, J., Hessels, J. W. T., et al. 2014, A\&A, 570, 60

Cordes, J. M., Lazio, T. J. W. 2002, preprint (arXiv:astro-ph/0207156)

Cordes, J. M., Rickett, B. J., Stinebring, D. R., Coles, W. A. 2006, ApJ, 637, 346

Deller, A. T., Boyles, J., Lorimer, D. R. et al., 2013, ApJ, 770, 145.

Deller, A. T., Brisken, W. F., Chatterjee, S., et al. 2011, 20th Meeting of EVGA, (Bonn, Germany, March 29-30, 2011), Eds: W. Alef, S. Bernhart, and A. Nothnagel, p. 178-182

Dewdney, P., Turner, W., Millenaar, R., McCool, R., Lazio, J., Cornwell, T., 2013, "SKA1 System Baseline Design", Document number SKA-TEL-SKO-DD-001 Revision 1

Ferriére, K., Terral, P. 2014, A\&A 561, 100

Finkbeiner, D. P., 2003, ApJS, 146, 407.

Gaensler, B. M., Madsen, G. J., Chatterjee, S. \& Mao, S. A. 2008, PASA, 25, 184

Green, J., van Langevelde, H. J., Brunthaler, A., et al., 2015, "Maser astrometry with VLBI and the SKA", in proc. Advancing Astrophysics with the Square Kilometre Array, PoS(AASKA14)119

Gupta, P., Rickett, B. J., Lyne, A. 1994, MNRAS, 269, 1035

Han, J. L., 2009, "Magnetic structure of our Galaxy: A review of observations", in proceedings of "Cosmic Magnetic Fields: From Planets, to Stars and Galaxies", IAU Symp. 259, 455

Han, J. L., 2013, "Pulsars as excellent probes for the magnetic structure in our Milky Way", in proceedings of "Neutron Stars and Pulsars: Challenges and Opportunities after 80 years", IAU Symp. 291, 223

Han, J. L., Ferriere, K., Manchester, R. N. 2004, ApJ, 610, 820.

Han, J. L., Manchester, R. N., Berkhuijsen, E. M., Beck, R. 1997, A\&A 322, 98

Han, J. L., Manchester, R. N., Lyne, A. G., Qiao, G. J., van Straten, W. 2006, ApJ, 642, 868.

Han, J. L., Manchester, Qiao, G. J. 1999, MNRAS, 306, 371.

Han, J. L., Zhang, J. S. 2007, A\&A, 464, 609

Harvey-Smith, L., Madsen, G. J., Gaensler, B. M. 2011, ApJ, 736, 83 
Haverkorn, M., Akahori, T., Carretti, E., et al., 2015, "Measuring magnetism in the Milky Way with the Square Kilometre Array", in proc. Advancing Astrophysics with the Square Kilometre Array, PoS(AASKA14)096

Heald, G., Beck, R., de Blok, W. J. G., et al., 2015, "Magnetic field tomography in nearby galaxies with the Square Kilometre Array", in proc. Advancing Astrophysics with the Square Kilometre Array, PoS(AASKA14) 106

Hou, L. G., Han, J. L., 2014, A\&A, 569, A125

Janssen, G. H., Hobbs, G., McLaughlin, M. et al., 2015, "Gravitational wave astronomy with the SKA", in proc. Advancing Astrophysics with the Square Kilometre Array, PoS(AASKA14)037

Johnston-Hollitt M., 2015, "Using SKA Rotation Measures to Reveal the Mysteries of the Magnetised Universe", in proc. Advancing Astrophysics with the Square Kilometre Array, PoS(AASKA14)092

Karastergiou, A., Johnston, S., Andersson, N., et al. 2015, "Understanding pulsar magnetospheres with the SKA", in proc. Advancing Astrophysics with the Square Kilometre Array, PoS(AASKA14)038

Keane, E. F., Bhattacharyya, B., Kramer, M., et al. 2015, "A Cosmic Census of Radio Pulsars with the SKA", in proc. Advancing Astrophysics with the Square Kilometre Array, PoS(AASKA14)040

Keith, M. J., et al. 2013, MNRAS, 429, 2161

Lazio, T. J. W., 2004, ApJ, 613, 1023

Lewandowski, W., Dembska, M., Kijak, J., Kowalinska, M., 2013, MNRAS, 434, 69

Löhmer, O., Kramer, M., Mitra, D., Lorimer, D. R., Lyne, A. G. 2001, ApJ, 562, L157

Löhmer, O., Mitra, D., Gupta, Y., Kramer, M., Ahuja, A. 2004, A\&A, 425, 569

Lorimer, D. R., Bailes, M., McLaughlin, M. A., et al. 2007, Science, 318, 777

Lyne, A. G., 1971, in Proceedings of IAU Symp. 46, p.182

Manchester, R. N., Fan, G., Lyne, A. G., et al. 2006, ApJ, 649, 235

Macquart J.-P., Keane, E. F., Grainge, K., et al. 2015, "Fast transients at cosmological distances", in proc. Advancing Astrophysics with the Square Kilometre Array, PoS(AASKA14)055

Manchester, R. N., Hobbs, G. B., Teoh, A., Hobbs, M., 2005, AJ, 129, 1993

McBride, J., Heiles, C., 2013, ApJ, 763, 8

Minter, A. H., Spangler, S. R., 1996, ApJ, 458, 194

Mitra, D., Wielebinski, R., Kramer, M., Jessner, A. 2003, A\&A, 398, 993

Nan, R., Li, D., Jin, C., et al., 2011, International Journal of Modern Physics D, 20, 989

Paragi, Z., Godfrey, L., Reynolds, C. et al, 2015, "Very Long Baseline Interferometry with the SKA", in proc. Advancing Astrophysics with the Square Kilometre Array, PoS(AASKA14)143

Pen, U.-L., Levin, Y., 2014, MNRAS 442, 3338

Pen, U.-L., Macquart, J.-P., Deller, A. T., Brisken W., 2014, MNRAS, 440, L36

Petroff, E., Keith, M. J., Johnston, S., van Straten, W., Shannon, R. M. 2013, MNRAS, 435, 1610

Prouza, M., Šmída, R. 2003, A\&A, 410, 1

Robishaw, T., Green, J. A., Surcis, G., et al., 2015, "Measuring Magnetic Fields Near and Far with the SKA via the Zeeman Effect", in proc. Advancing Astrophysics with the Square Kilometre Array, PoS(AASKA14) 110

Robishaw, T., Quataert, E., Heiles, C., 2008, ApJ, 680, 981 
Savage, A. H., Spangler, S. R., \& Fischer, P. D. 2013, ApJ, 765, 42

Schnitzeler, D. H. F. M 2012, MNRAS, 427, 664

Smits, R., Tingay, S. J., Wex, N., Kramer, M., Stappers, B. 2011, A\&A, 528, 108

Sotomayor-Beltran, C., Sobey, C., Hessels, J. W. T., et al. 2013, A\&A, 552, A58

Spitler, L. G., Cordes, J. M., Hessels, J. W. T., Lorimer, D. R., et al. 2014a, ApJ, 790, 101

Spitler, L. G., Lee, K. J., Eatough, R. P., et al. 2014b, ApJ, 780, L3

Stinebring, D. R., 2006, ChJAS, Vol.6, Issue S2, 204

Sun, X. H., Reich, W., Waelkens, A., Enßlin, T. A. 2008, A\&A 477, 573

Sun, X. H., Reich, W., Wang, C., Han, J. L., Reich, P. 2011, A\&A 535, A64

Taylor, J. H., Cordes, J. M., 1993, ApJ, 411, 674

Thornton, D., Stappers, B., Bailes, M., et al. 2013, Science, 341, 53

Xu, J., Han, J. L, 2014a, MNRAS, 442, 3329

Xu, J., Han, J. L, 2014b, RAA, 14, 942

You, X. P., Hobbs, G., Coles, W. A., et al. 2007, MNRAS, 378, 493

You, X. P., Coles, W. A., Hobbs, G., Manchester, R. N. 2012, MNRAS, 422, 1160 